\title{
A New Membership Function on Hexagonal Fuzzy Numbers
}

\author{
K. Dhurai ${ }^{1}$, A. Karpagam ${ }^{2}$ \\ ${ }^{1}$ Assistant Professor, Department of Mathematics, Valliammai Engineering College, Kattankulathur-603203, India
}

\begin{abstract}
In this paper, a new membership function to introduce on Hexagonal Fuzzy numbers which defines where the methods of addition, subtraction and multiplication has been modified with some conditions. The main aim of this paper is to introduce a new membership function and satisfies the operation of addition, subtraction and multiplication of hexagonal fuzzy number on the basis of alpha cut sets of fuzzy numbers.
\end{abstract}

Keywords: Membership function, Fuzzy Arithmetic, Hexagonal Fuzzy number, Alpha cut

\section{Introduction}

The concept of fuzzy logic was first conceived by Lotfi.A. Zadeh (1965). Fuzzy set theory permits the gradual assessment of the membership of elements in a set which is described in the interval $[0,1]$. It can be used in a wide range of domains where information is incomplete and imprecise. In this paper a new membership function of Hexagonal Fuzzy numbers has been introduced with its basic membership function followed by the arithmetic operations of fuzzy numbers.

In section 2 preliminaries and section 3 the new membership function are discussed with numerical example and section 4 concludes the paper.

\section{Preliminaries}

\subsection{Definition}

The characteristic function $\mu_{\mathrm{A}}$ of a crisp set A C X assigns a value either 0 or 1 to each member in $\mathrm{X}$. This function can be generalized to a function $\mu_{\tilde{A}}$ such that the value assigned to the element of the universal set $\mathrm{X}$ fall within a specified range i.e. $\mu_{\tilde{A}}: \mathrm{X} \rightarrow[0,1]$. The assigned value indicate the membership function and the set $\widetilde{A}=\left\{\left(\mathrm{x}, \mu_{\widetilde{A}}(x)\right) ; \mathrm{x} \in \mathrm{X}\right\}$ defined by $\mu_{\tilde{A}}(x)$ for $\mathrm{x} \epsilon \mathrm{X}$ is called fuzzy set.

\subsection{Definition}

A fuzzy number $\widetilde{A_{H}}$ is a hexagonal fuzzy number denoted by $\widetilde{A_{H}}=\left(a_{1}, a_{2}, a_{3}, a_{4}, a_{5}, a_{6}\right)$ where $\left(a_{1}, a_{2}, a_{3}, a_{4}, a_{5}, a_{6}\right)$ are real numbers and its membership function $\mu_{\overparen{A_{H}}}(x)$ is given below.

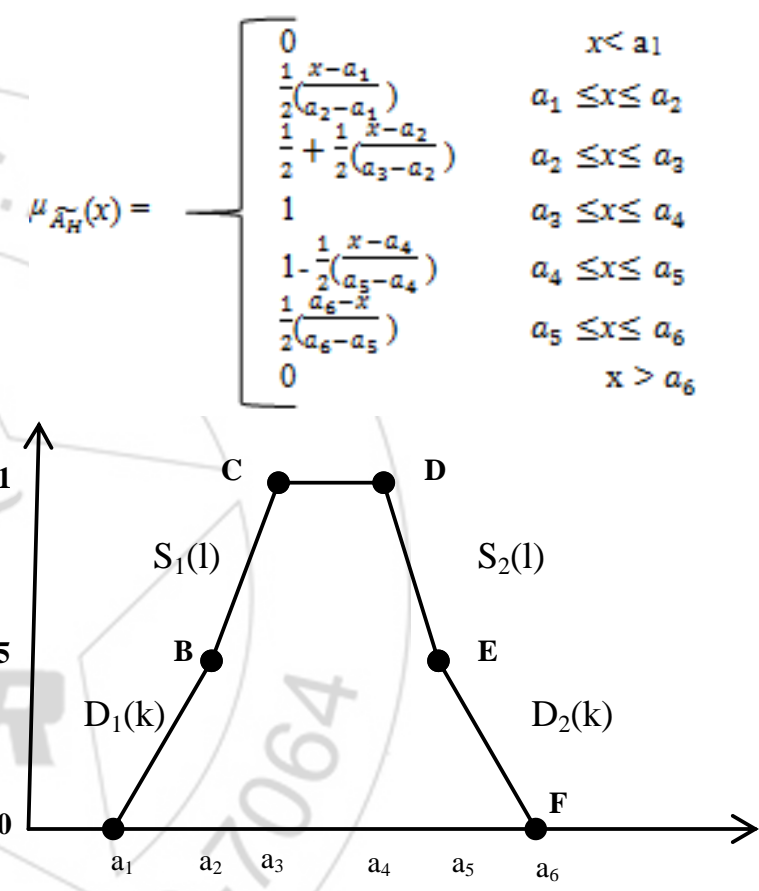

Figure1: Graphical representation of a hexagonal fuzzy number for $\mathrm{x} \in[0,1]$

\subsection{Definition}

An Hexagonal fuzzy number denoted by $\widetilde{A_{H}}$ is defined as ( $\left(D_{1}(k), S_{1}(l), S_{2}(l), D_{2}(k)\right)$ for $\mathrm{k} \in[0,0.5]$ and $l \in[0.5,1]$ where,

(i) $\left(D_{1}(k)\right.$ is a bounded left continuous non decreasing function over $[0,0.5]$

(ii) $S_{1}(l)$ is a bounded left continuous non decreasing function over $[0.5,1]$

(iii) $S_{2}(l)$ is a bounded left continuous non increasing function over [1, 0.5]

(iv) $D_{2}(k)$ is a bounded left continuous non increasing function over $[0.5, \mathrm{o}]$

\section{New Membership Function}

A fuzzy number $\widetilde{A_{H}}$ is a hexagonal fuzzy number denoted by $\widetilde{A_{H}}=\left(a_{1}, a_{2}, a_{3}, a_{4}, a_{5}, a_{6}\right)$ where $\left(a_{1}, a_{2}, a_{3}, a_{4}, a_{5}, a_{6}\right)$ 


\section{International Journal of Science and Research (IJSR) \\ ISSN (Online): 2319-7064}

Index Copernicus Value (2013): 6.14 | Impact Factor (2015): 6.391

are real numbers and its membership function $\mu_{\widetilde{A_{H}}}(x)$ is given below.

$$
\mu_{A_{H}}(x)=\left\{\begin{array}{cc}
0 & x<a_{1} \\
\left(\frac{x-a_{1}}{a_{3}-a_{1}}\right) & a_{1} \leq x \leq \frac{a_{1}+a_{3}}{2} \\
\frac{1}{2}+\frac{1}{2}\left(\frac{2 x-a_{1}-a_{3}}{a_{3}-a_{1}}\right) & \frac{a_{1}+a_{3}}{2} \leq x \leq a_{3} \\
1 & a_{3} \leq x \leq a_{4} \\
1-\left(\frac{x-a_{4}}{a_{6}-a_{4}}\right) & a_{4} \leq x \leq \frac{a_{4}+a_{6}}{2} \\
\left(\frac{x-a_{6}}{a_{4}-a_{6}}\right) & \frac{a_{4}+a_{6}}{2} \leq x \leq a_{6} \\
0 & x>a_{6}
\end{array}\right.
$$

\subsection{Alpha Cut}

The classical set $\widetilde{A_{\alpha}}$ called alpha cut set is the set of elements whose degree of membership is the set of elements whose degree of membership in $\widetilde{A_{H}}=\left(a_{1}, a_{2}, a_{3}, a_{4}, a_{5}, a_{6}\right.$ ) is no less than, $\alpha$ it is defined as

$$
\begin{aligned}
& A_{\alpha}=\left\{\left\{\mathrm{x} \in \mathrm{X} / \mu_{\tilde{A}_{H}}(x) \geq \alpha\right\}\right.
\end{aligned}
$$

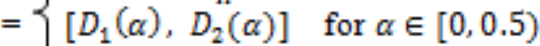

$$
\begin{aligned}
& {\left[S_{1}(\alpha), S_{2}(\alpha)\right] \text { for } \alpha \in[0.5,1]}
\end{aligned}
$$

\section{$3.2 \alpha$ cut operations}

If we get crisp interval by $\alpha$ cut operations interval $A_{\alpha}$ shall be obtained as follows for all $\alpha \in[0,1]$

Consider $S_{1}(x)=\alpha$

$\frac{1}{2}+\frac{1}{2}\left(\frac{2 x-a_{1}-a_{3}}{a_{3}-a_{1}}\right)=\alpha$

$\mathrm{x}=\alpha\left(a_{3}-a_{1}\right)+a_{1}$

(i e) $S_{1}(\alpha)=\alpha\left(a_{3}-a_{1}\right)+a_{1}$

Similarly from $S_{2}(x)=\alpha$

$1-\left(\frac{x-a_{4}}{a_{6}-a_{4}}\right)=\alpha$

(i e) $S_{2}(\alpha)=a_{6}-\alpha\left(a_{6}-a_{4}\right)$

This implies $\left[S_{1}(\alpha), S_{2}(\alpha)\right]=\left[\alpha\left(a_{3}-a_{1}\right)+a_{1}, a_{6}-\right.$ $\left.\alpha\left(a_{6}-a_{4}\right)\right]$

Consider $D_{1}(x)=\alpha$

$D_{1}(\alpha)=\mathrm{x}=a_{1}+\alpha\left(a_{3}-a_{1}\right)$

Similarly from $D_{2}(x)=\alpha$, we get

$$
D_{2}(\alpha)=\mathrm{x}=a_{6}+\alpha\left(a_{4}-a_{6}\right)
$$

This implies $\left[D_{1}(\alpha), D_{2}(\alpha)\right]=\left[a_{1}+\alpha\left(a_{3}-a_{1}\right)+\right.$, $\left.a_{6}+\alpha\left(a_{4}-a_{6}\right)\right]$

Hence

$\boldsymbol{A}_{\alpha}$ $=\left\{\begin{array}{c}{\left[a_{1}+\alpha\left(a_{3}-a_{1}\right), a_{6}+\alpha\left(a_{4}-a_{6}\right) \text { for } \alpha \in[0,0.5)\right.} \\ {\left[\alpha\left(a_{3}-a_{1}\right)+a_{1}, \quad a_{6}-\alpha\left(a_{6}-a_{4}\right)\right] \text { for } \alpha \in[0.5,1]}\end{array}\right.$

\subsection{Operations of Hexagonal Fuzzy numbers}

The three operations that can be performed on hexagonal fuzzy numbers, suppose $\widetilde{A_{H}}=\left(a_{1}, a_{2}, a_{3}, a_{4}, a_{5}, a_{6}\right)$ and $\widetilde{B_{H}}=\left(b_{1}, b_{2}, b_{3}, b_{4}, b_{5}, b_{6}\right)$ are two hexagonal fuzzy numbers then

(i) Addition: $\widetilde{A_{H}}+\widetilde{B_{H}}=\left(a_{1}+b_{1}, a_{2}+b_{2}, a_{3}+b_{3}, a_{4}+\right.$ $\left.b_{4}, a_{5}+b_{5}, a_{6}+b_{6}\right)$

(ii) Subtraction: $\widetilde{A_{H}}-\widetilde{B_{H}}=\left(a_{1}-b_{1}, a_{2}-b_{2}, a_{3}-\right.$ $\left.b_{3}, a_{4}-b_{4}, a_{5}-b_{5}, a_{6}-b_{6}\right)$

(iii) Multiplication: $\widetilde{A_{H}}(*) \widetilde{B_{H}}=\left(a_{1} * b_{1}, a_{2} * b_{2}, a_{3} *\right.$

$\left.b_{3}, a_{4} * b_{4}, a_{5} * b_{5}, a_{6} * b_{6}\right)$
Example 1:

Let $\widetilde{A_{H}}=(1,2,3,5,6,7)$ and $\widetilde{B_{H}}=(2,4,6,8,10,12)$ are two hexagonal fuzzy numbers then

$\widetilde{A_{H}}+\widetilde{B_{H}}=(3,6,9,13,16,19)$

Example 2:

Let $\widetilde{A_{H}}=(1,2,3,5,6,7)$ and $\widetilde{B_{H}}=(2,4,6,10,12,14)$ are two hexagonal fuzzy numbers then

$\widetilde{A_{H}}-\widetilde{B_{H}}=(-1,-2,-3,-5,-6,-7)$

Example 3:

Let $\widetilde{A_{H}}=(1,2,3,5,6,7)$ and $\widetilde{B_{H}}=(2,4,6,8,10,12)$ are two hexagonal fuzzy numbers then

$\widetilde{A_{H}}(*) \widetilde{B_{H}}=(2,8,18,40,60,84)$

\subsection{Operations for Addition, Subtraction \& Multiplication on Hexagonal Fuzzy Number}

\subsection{1 $\alpha$ cut of a normal hexagonal fuzzy number}

The $\alpha$ cut of a normal hexagonal fuzzy number $\widetilde{A_{H}}=\left(a_{1}\right.$, $\left.a_{2}, a_{3}, a_{4}, a_{5}, a_{6}\right)$ given by the definition for all $\alpha \in[0,1]$

$\boldsymbol{A}_{\boldsymbol{\alpha}}$

$=\left\{\left[a_{1}+\alpha\left(a_{3}-a_{1}\right), a_{6}+\alpha\left(a_{4}-a_{6}\right)\right.\right.$ for $\alpha \in[0,0.5)$

$= \begin{cases}\alpha\left(a_{3}-a_{1}\right)+a_{1}, & \left.a_{6}-\alpha\left(a_{6}-a_{4}\right)\right] \text { for } \alpha \in[0.5,1]\end{cases}$

\subsubsection{Addition of Two hexagonal fuzzy numbers}

Let $\widetilde{A_{H}}=\left(a_{1}, a_{2}, a_{3}, a_{4}, a_{5}, a_{6}\right)$ and $\widetilde{B_{H}}=\left(b_{1}, b_{2}, b_{3}, b_{4}, b\right.$ ,$\left.b_{6}\right)$ are two hexagonal fuzzy numbers for all $\alpha \in[0,1]$. Let us add the alpha cuts $A_{\alpha}$ and $B_{\alpha}$ of $\widetilde{A_{H}}$ and $\widetilde{B_{H}}$ using interval arithmetic.

$\boldsymbol{A}_{\boldsymbol{\alpha}}+\boldsymbol{B}_{\boldsymbol{\alpha}}$

$=\left\{\begin{array}{c}{\left[a_{1}+\alpha\left(a_{3}-a_{1}\right), a_{6}+\alpha\left(a_{4}-a_{6}\right)\right]} \\ +\left[b_{1}+\alpha\left(b_{3}-b_{1}\right), b_{6}+\alpha\left(b_{4}-b_{6}\right)\right] \text { for } \alpha \in[0,0.5) \\ {\left[\alpha\left(a_{3}-a_{1}\right)+a_{1}, a_{6}-\alpha\left(a_{6}-a_{4}\right)\right]+} \\ {\left[\alpha\left(b_{3}-b_{1}\right)+b_{1}, \quad b_{6}-\alpha\left(b_{6}-b_{4}\right)\right] \text { for } \alpha \in[0.5,1]}\end{array}\right.$

Consider the example 1 such that

For $\alpha \in[0,0.5)$

$A_{\alpha}=[2 \alpha+1,-2 \alpha+7] \quad B_{\alpha}=[4 \alpha+2,-4 \alpha+12]$

$A_{\alpha}+B_{\alpha}=[6 \alpha+3,-6 \alpha+19]$

For $\alpha \in[0.5,1]$

$A_{\alpha}=[2 \alpha+1,-2 \alpha+7] \quad B_{\alpha}=[4 \alpha+2,-4 \alpha+12]$

$A_{\alpha}+B_{\alpha}=[6 \alpha+3,-6 \alpha+19]$

Since for both $\alpha \in[0,0.5) \& \alpha \in[0.5,1]$ arithmetic intervals are same.

Therefore $A_{\alpha}+B_{\alpha}=[6 \alpha+3,-6 \alpha+19]$ for all $\alpha \in[0,1]$

When $\alpha=0, A_{0}+B_{0}=[3,19]$

$\alpha=0.5, A_{0.5}+B_{0.5}=[6,16]$

And for $\alpha=1, A_{1}+B_{1}=[9,13]$, Hence $A_{\alpha}+B_{\alpha}=[3,6,9$, $13,16,19]$ hence all the points coincide with the sum of the two hexagonal fuzzy number.

Therefore addition of two $\alpha$ cuts lies within the interval.

\subsubsection{Subtraction of Two hexagonal fuzzy numbers:}

Let $\widetilde{A_{H}}=\left(a_{1}, a_{2}, a_{3}, a_{4}, a_{5}, a_{6}\right)$ and $\widetilde{B_{H}}=\left(b_{1}, b_{2}, b_{3}, b_{4}, b\right.$ ,$\left.b_{6}\right)$ are two hexagonal fuzzy numbers for all $\alpha \in[0,1]$. Let us subtract the alpha cuts $A_{\alpha}$ and $B_{\alpha}$ of $\widetilde{A_{H}}$ and $\widetilde{B_{H}}$ using interval arithmetic. 


\section{International Journal of Science and Research (IJSR) \\ ISSN (Online): 2319-7064}

Index Copernicus Value (2013): 6.14 | Impact Factor (2015): 6.391

$\boldsymbol{A}_{\boldsymbol{\alpha}}-\boldsymbol{B}_{\boldsymbol{\alpha}}$
$=\left\{\begin{array}{c}{\left[a_{1}+\alpha\left(a_{3}-a_{1}\right), a_{6}+\alpha\left(a_{4}-a_{6}\right)\right]} \\ -\left[b_{1}+\alpha\left(b_{3}-b_{1}\right), b_{6}+\alpha\left(b_{4}-b_{6}\right)\right] \text { for } \alpha \in[0,0.5) \\ {\left[\alpha\left(a_{3}-a_{1}\right)+a_{1}, a_{6}-\alpha\left(a_{6}-a_{4}\right)\right]} \\ -\left[\alpha\left(b_{3}-b_{1}\right)+b_{1}, \quad b_{6}-\alpha\left(b_{6}-b_{4}\right)\right] \text { for } \alpha \in[0.5,1]\end{array}\right.$

Consider the example 2 such that

For $\alpha \in[0,0.5)$

$A_{\alpha}-B_{\alpha}=[-2 \alpha-1,2 \alpha-7]$

For $\alpha \in[0.5,1]$

$A_{\alpha}-B_{\alpha}=[-2 \alpha-1,2 \alpha-7]$

Since for both $\alpha \in[0,0.5) \& \alpha \in[0.5,1]$ arithmetic intervals are same.

Therefore $A_{\alpha}-B_{\alpha}==[-2 \alpha-1,2 \alpha-7]$ for all $\alpha \in[0,1]$

When $\alpha=0, A_{0}-B_{0}=[-1,-7]$

$\alpha=0.5, \quad A_{0.5}-B_{0.5}=[-3,-5]$

And for $\alpha=1, A_{1}-B_{1}=[-2,-6]$, Hence $A_{\alpha}-B_{\alpha}=[-1,-2$, -

$3,-5,-6,-7]$ hence all the points coincide with the difference

of the two hexagonal fuzzy number.

Therefore difference of two $\alpha$ cuts lies within the interval.

\subsubsection{Multiplication of Two hexagonal fuzzy numbers}

Let $\widetilde{A_{H}}=\left(a_{1}, a_{2}, a_{3}, a_{4}, a_{5}, a_{6}\right)$ and $\widetilde{B_{H}}=\left(b_{1}, b_{2}, b_{3}, b_{4}, b\right.$ ,$\left.b_{6}\right)$ are two hexagonal fuzzy numbers for all $\alpha \in[0,1]$. Let us multiply the alpha cuts $A_{\alpha}$ and $B_{\alpha}$ of $\widetilde{A_{H}}$ and $\widetilde{B_{H}}$ using interval arithmetic.

$$
\begin{gathered}
\boldsymbol{A}_{\boldsymbol{\alpha}} * \boldsymbol{B}_{\boldsymbol{\alpha}} \quad\left[a_{1}+\alpha\left(a_{3}-a_{1}\right), a_{6}+\alpha\left(a_{4}-a_{6}\right)\right] \\
=\left\{\begin{array}{c}
*\left[b_{1}+\alpha\left(b_{3}-b_{1}\right), b_{6}+\alpha\left(b_{4}-b_{6}\right)\right] \text { for } \alpha \in[0,0.5) \\
{\left[\alpha\left(a_{3}-a_{1}\right)+a_{1}, a_{6}-\alpha\left(a_{6}-a_{4}\right)\right] *} \\
{\left[\alpha\left(b_{3}-b_{1}\right)+b_{1}, \quad b_{6}-\alpha\left(b_{6}-b_{4}\right)\right] \text { for } \alpha \in[0.5,1]}
\end{array}\right.
\end{gathered}
$$

Consider the example 3 such that

For $\alpha \in[0,0.5)$

$A_{\alpha} * B_{\alpha}=[(2 \alpha+1)(4 \alpha+2),(-2 \alpha+7)(-4 \alpha+12)]$

For $\alpha \in[0.5,1]$

$A_{\alpha} * B_{\alpha}=[(2 \alpha+1)(4 \alpha+2),(-2 \alpha+7)(-4 \alpha+12)]$

Since for both $\alpha \in[0,0.5) \& \alpha \in[0.5,1]$ arithmetic intervals are same.

Therefore $A_{\alpha} * B_{\alpha}=[(2 \alpha+1)(4 \alpha+2),(-2 \alpha+7)(-4 \alpha+12)]$

for all $\alpha \in[0,1]$

When $\alpha=0, A_{0} * B_{0}=[2,84]$

$\alpha=0.5, \quad A_{0.5} * B_{0.5}=[8,60]$

And for $\alpha=1, \quad A_{1} * B_{1}=[18,40]$, Hence $A_{\alpha} * B_{\alpha}=[2,8$,

$18,40,60,84]$ hence all the points coincide with the product of the two hexagonal fuzzy number.

Therefore multiplication of two $\alpha$ cuts lies within the interval

\section{Conclusion}

In this paper, Membership function of hexagonal fuzzy number has been newly introduced and the alpha cut operations of arithmetic function principles using addition, subtraction and multiplication has been fully modified with some conditions and has been explained with numerical examples. We have obtained the same results from both the existing membership function as well as the proposed membership function. If the number of variables and parameters are reduced, this method will be far more efficient and easy when compared to the earlier method.

\section{References}

[1] Amit kumar, Jagdeep kaur and Pushpinder sign, "Fuzzy optimal solution of fully fuzzy linear programming problems with inequality constraints," International Journal of mathematical and computer science, pp. 37-40, 2010.

[2] Abhinav Bansal,"Trapezoidal Fuzzy numbers: Arithmetic behavior, International journal of physical and Mathematical Sciences, ISSN N0 2010-1791, 2011.

[3] A.Bansal, "Some non linear arithmetic operations on triangular fuzzy numbers," Advances in fuzzy mathematics, 5, pp. 147-156, 2010.

[4] D.Dubois and H.Prade, "Operations on fuzzy numbers," International journal of systems science, vol.9, no.6, pp.613-626, 1978.

[5] P.S.Dwyer, "Fuzzy sets, Information and Control," no.8, pp.338-353, 1965.

[6] S. Heilpern, "Representation and application of fuzzy numbers," Fuzzy sets and systems, vol.91, no.2., pp.259-268, 1997.

[7] "Klir, "Fuzzy arithmetic with requisite constraints," Fuzzy sets system, vol.91, pp.165-175, 1997.

[8] A.Kauffmann, M.Gupta,"Introduction to fuzzy Arithmetic," Theory and applications, Van Nostrand Reinhold, New York, 1980.

[9] S.U.Malini, Felbin.C.Kennedy, “An approach for solving fuzzy Transportation using octagonal Fuzzy numbers," Applied Mathematical Sciences, No.54, pp.2661-2673, 2013.

[10] P.Rajarajeswari, A.Sahaya Sudha, and R.Karthika, “ A New Operation on Hexagonal Fuzzy Number," International Journal of fuzzy Logic Systems, vol.3, No.3, pp-15-26, 2013.

[11] S. Rezvani, "Multiplication operation on Trapezoidal Fuzzy numbers," Journal of Physical sciences, vol. no. 15, pp.17-26, 2011.

[12] L.A.Zadeh, "Fuzzy Set," Information and Control, No.8, pp-338-353, 1965.

[13] H.J.Zimmermann, "Fuzzy Set theory and its applications," third edition, Klue Academic publishers, Bosten Massachusetts 1996. 